\title{
Treatment recommendations to cancer patients in the context of FDA guidance for next generation sequencing
}

Grace K. Dy ${ }^{1}$, Mary K. Nesline ${ }^{2}$, Antonios Papanicolau-Sengos², Paul DePietro², Charles M. LeVea ${ }^{3}$, Amy Early', Hongbin Chen', Anne Grand'Maison'1, Patrick Boland ${ }^{1}$, Marc S. Ernstoff', Stephen Edge ${ }^{8}$, Stacey Akers', Mateusz Opyrchal', Gurkamal Chatta', Kunle Odunsi', Sarabjot Pabla'2, Jeffrey M. Conroy ${ }^{2,4}$, Sean T. Glenn ${ }^{2,5}$, Hanchun T. DeFedericis², Blake Burgher ${ }^{2}$, Jonathan Andreas², Vincent Giamo², Maochun Qin², Yirong Wang ${ }^{2}$, Kazunori Kanehira ${ }^{3}$, Felicia L. Lenzo ${ }^{2}$, Peter Frederick ${ }^{6}$, Shashikant Lele ${ }^{6}$, Lorenzo Galluzzi ${ }^{7,8,9}$, Boris Kuvshinoff ${ }^{10}$ and Carl Morrison ${ }^{2,3,5^{*}}$ (D)

\begin{abstract}
Background: Regulatory approval of next generation sequencing (NGS) by the FDA is advancing the use of genomicbased precision medicine for the therapeutic management of cancer as standard care. Recent FDA guidance for the classification of genomic variants based on clinical evidence to aid clinicians in understanding the actionability of identified variants provided by comprehensive NGS panels has also been set forth. In this retrospective analysis, we interpreted and applied the FDA variant classification guidance to comprehensive NGS testing performed for advanced cancer patients and assessed oncologist agreement with NGS test treatment recommendations.
\end{abstract}

Methods: NGS comprehensive genomic profiling was performed in a CLIA certified lab (657 completed tests for 646 patients treated at Roswell Park Comprehensive Cancer Center) between June 2016 and June 2017. Physician treatment recommendations made within 120 days post-test were gathered from tested patients' medical records and classified as targeted therapy, precision medicine clinical trial, immunotherapy, hormonal therapy, chemotherapy/radiation, surgery, transplant, or non-therapeutic (hospice, surveillance, or palliative care). Agreement between NGS test report targeted therapy recommendations based on the FDA variant classification and physician targeted therapy treatment recommendations were evaluated.

Results: Excluding variants contraindicating targeted therapy (i.e., KRAS or NRAS mutations), at least one variant with FDA level 1 companion diagnostic supporting evidence as the most actionable was identified in 14\% of tests, with physicians most frequently recommending targeted therapy (48\%) for patients with these results. This stands in contrast to physicians recommending targeted therapy based on test results with FDA level 2 (practice guideline) or FDA level 3 (clinical trial or off label) evidence as the most actionable result (11 and 4\%, respectively).

Conclusions: We found an appropriate "dose-response" relationship between the strength of clinical evidence supporting biomarker-directed targeted therapy based on application of FDA guidance for NGS test variant classification, and subsequent treatment recommendations made by treating physicians. In view of recent changes at FDA, it is paramount to define regulatory grounds and medical policy coverage for NGS testing based on this guidance.

Keywords: Next generation sequencing, Comprehensive genomic profiling, FDA guidance, Physician treatment recommendations

\footnotetext{
* Correspondence: Carl.Morrison@Omniseq.com

${ }^{2}$ OmniSeq, Inc., Buffalo, NY 14203, USA

${ }^{3}$ Department of Pathology, Roswell Park Comprehensive Cancer Center,

Buffalo, NY 14263, USA

Full list of author information is available at the end of the article
}

(c) The Author(s). 2019 Open Access This article is distributed under the terms of the Creative Commons Attribution 4.0 International License (http://creativecommons.org/licenses/by/4.0/), which permits unrestricted use, distribution, and reproduction in any medium, provided you give appropriate credit to the original author(s) and the source, provide a link to the Creative Commons license, and indicate if changes were made. The Creative Commons Public Domain Dedication waiver (http://creativecommons.org/publicdomain/zero/1.0/) applies to the data made available in this article, unless otherwise stated. 


\section{Background}

The Food and Drug Administration (FDA) has announced new mechanisms for regulatory approval of next generation sequencing (NGS) [1]. This includes a new pathway for approval of NGS tests for tumor profiling using the New York State Department of Health (NYSDOH) as a FDA third-party reviewer of in vitro diagnostics [2]. Central to this review is the fundamental tenet that NGS tests cover biomarkers with predictive value that change over time as clinical and scientific discoveries are made. As such, the FDA now recognizes three evidence levels that support the actionability and clinical utility of NGS tests [1]. Level 1 variants are defined by FDA as essential for the safe and effective use of a corresponding therapeutic. In seeking FDA approval, NGS tests may include level 1 evidence claims for a specific drug based on support for the analytical validity of the test for each specific biomarker and a clinical study that establishes either the link between the result of that test and patient outcomes or the clinical concordance to a previously approved companion diagnostic. Level 2 variants are defined by FDA as enabling physicians to use information about their patients in accordance with supporting clinical evidence, such as professional guidelines and/or peer-reviewed publications. Level 3 variants are defined by FDA as informational or used to direct patients toward clinical trials. Such claims are supported by analytical validation, principally through a representative approach when appropriate, and clinical or mechanistic rationale for inclusion in the panel, including peer-reviewed publications or in vitro preclinical models. While many groups have implemented other classification approaches for disease-biomarker-drug evidence associations, the FDA's approach uniquely focuses on NGS and explicitly requires the analytical validity of the gene-variants tested.

Not unlike many other major cancer-focused medical academic centers [3-10], Roswell Park Comprehensive Cancer Center (Buffalo, NY) developed a precision oncology initiative that included NGS tests approved by the New York State Department of Health (NYSDOH). In 2016, an NGS pan-cancer comprehensive genomic profiling panel called OmniSeq Comprehensive ${ }^{\bullet}$ (OCP) (OmniSeq ${ }^{\oplus}$, Buffalo, NY) was launched, based upon the Oncomine $^{\mathrm{Tm}}$ Comprehensive Assay by ThermoFisher Scientific (Carpinteria, CA). To evaluate the utility of the above described FDA variant classification guidance for oncologist treatment recommendations, we interpreted and applied the three-tier evidence schema to OCP test results from a large cohort of sequentially tested advanced cancer patients, and assessed agreement between NGS test targeted therapy recommendations and subsequent physician treatment recommendations for their patients.

\section{Methods}

The OCP test uses tumor tissue to detect all classes of somatic genomic alterations in 144 cancer-associated genes. As seen in Table 1, the DNA-Seq component of the test detects somatic mutations (single nucleotide variants, insertions and deletions) and copy number variants in both oncogenes and tumor suppressor genes, while the RNA-Seq component performs rearrangement (fusion) analysis in oncogenes. DNA mutational analysis requires a minimum depth of 457 reads and uses a hot spot coverage strategy to detect gain-of-function mutations in oncogenes, while copy number analysis detects high level amplification. DNA mutational analysis also detects loss-of-function mutations in tumor suppressor genes using a complete coding sequence coverage strategy, while copy number analysis detects homozygous deletions. The RNA analysis detects fusions. The OCP test is approved for clinical use by New York State Clinical Laboratory Evaluation Program (NYS CLEP), which requires orthogonal confirmation by secondary technologies for somatic mutations. A proprietary bioinformatics pipeline filters single nucleotide polymorphisms and identifies reportable variants, including variants of unknown therapeutic significance (VUTS), based on pathogenicity using multiple public genomic content sources such as COSMIC, 1000 Genomes Project, dbSNP, SIFT, PolyPhen, and ClinVar.

Filtered, detected variants are submitted to a comprehensive knowledgebase of therapeutic associations, which determines whether or not there are therapeutic associations at the nucleotide, codon, exon, gene, or fusion level. A laboratory information engine with reporting rules specific to oncogenes and tumor suppressor genes to determine clinical significance in the final report. Variants in tumor suppressor genes must be pathogenic or deleterious by both SIFT and PolyPhen to be reported. While OCP does not sequence matching non-tumor tissue from tested patients, it is possible that germline mutations can be identified from tumor-only sequencing results without direct analysis of germline DNA. OCP reports detected mutations in genes prescribed by the American College of Medical Genetics and Genomics (ACMG) [11] as potentially hereditary, and directs physicians to further investigate by germline testing if clinically applicable. OCP test performance characteristics were analytically validated by OmniSeq Laboratories under the requirements of the Clinical Laboratory Improvement Amendments (CLIA) of 1988, and OmniSeq, Inc. is licensed by CLIA, College of American Pathologists (CAP), and the NYS CLEP to perform high-complexity molecular diagnostic testing. As such, OCP meets the analytical requirements put forth in the FDA variant classification guidelines for NGS testing. Additional details regarding OCP methodology, clinical 
Table 1 Gene-variants tested by OmniSeq Comprehensive ${ }^{\circledast}$

\begin{tabular}{ll}
\hline Variant Type & Genes \\
\hline Single Nucleotide Variants (SNVs), Insertions, & Hotspot: ABL1, AKT1, ALK, AR, ARAF, BRAF, BTK, CBL, CDK4, CHEK2, \\
Deletions, and Indels & CSF1R, CTNNB1, DDR2, DNMT3A, EGFR, ERBB2, ERBB3, ERBB4, ESR1, \\
& EZH2, FGFR1, FGFR2, FGFR3, FLT3, FOXL2, GATA2, GNA11, GNAQ, \\
& GNAS, HNF1A, HRAS, IDH1, IDH2, IFITM1, IFITM3, JAK1, JAK2, JAK3, \\
& KDR, KIT, KNSTRN, KRAS, MAGOH, MAP2K1, MAP2K2, MAPK1, MAX, \\
& MED12, MET, MLH1, MPL, MTOR, MYD88, NFE2L2, NPM1, NRAS, \\
& PAX5, PDGFRA, PIK3CA, PPP2R1A, PTPN11, RAC1, RAF1, RET, RHEB, \\
& RHOA, SF3B1, SMO, SPOP, SRC, STAT3, U2AF1, XPO1 \\
& Full Coding: APC, ATM, BAP1, BRCA1, BRCA2, CDH1, CDKN2A, \\
& FBXW7, GATA3, MSH2, NF1, NF2, NOTCH1, PIK3R1, PTCH1, PTEN, \\
& RB1, SMAD4, SMARCB1, STK11, TET2, TP53, TSC1, TSC2, VHL, WT1 \\
& ACVRL1, AKT1, APEX1, AR, ATP11B, BCL2L1, BCL9, BIRC2, BIRC3, \\
& CCND1, CCNE1, CD274, CD44, CDK4, CDK6, CSNK2A1, DCUN1D1, \\
& EGFR, ERBB2, FGFR1, FGFR2, FGFR3, FGFR4, FLT3, GAS6, IGF1R \\
& APC, ATM, BAP1, BRCA1, BRCA2, CDH1, CDKN2A, FBXW7, GATA3, \\
Copy Number Gain & MSH2, NF1, NF2, NOTCH1, PIK3R1, PTCH1, PTEN, RB1, SMAD4, \\
& SMARCB1, STK11, TET2, TP53, TSC1, TSC2, VHL, WT1 \\
& ABL1, AKT3, ALK, AXL, BRAF, EGFR, ERBB2, ERG, ETV1, ETV4, ETV5, \\
& FGFR1, FGFR2, FGFR3, MET, NTRK1, NTRK2, NTRK3, PDGFRA, PPARG, \\
\hline Fusions & RAF1, RET, ROS1 \\
\hline
\end{tabular}

validity, and performance characteristics can be found in the National Center for Biotechnology Information (NCBI) Genetic Testing Registry (https://www.ncbi.nlm. nih.gov/gtr/tests/552042/overview/).

Each gene-variant detected and reported by OCP tests previously performed between June 2017 to June 2017 ( $n=657$ ) was mapped to one or more levels of evidence based on interpretation of the FDA guidance for actionable variant classification, as follows: Level 1: variants listed on current FDA and/or European Medical Association (EMA) approved targeted therapy labels required for drug administration; Level 2: variants described in publicly available professional practice guidelines described as having evidence of response, resistance, or non-response to targeted therapeutics. Evidence sources to describe level 2 variants included professional practice guidelines established by the National Comprehensive Cancer Network (NCCN), and the European Society for Medical Oncology (ESMO). A complete list of level 1 and 2 therapeutic variant associations is shown in Table 2. Level 3: variants used as inclusion criteria or direct therapeutic targets of agents in active clinical development. Automated and manual review of investigational studies at https://clinicaltrials.gov was used for the identification of level 3 variants. As of this writing, there were 160 unique targeted agents, in use alone or in combination in over 350 recruiting precision medicine trials with OCP variants that act as direct therapeutic targets of the investigational agent, inclusion criteria, or both. Off-label use of variants listed on current FDA and/or EMA approved targeted therapy drug labels required for administration qualify were also defined as level 3 evidence since many targeted therapy trials are engaged in expanding existing indications to other tumor types.

Age, gender, stage of disease, and tumor type were retrieved from test requisition data. Medical records were reviewed for patients with at least 60 days of post-test follow up time available in order to collect treatment status at the time of test order and physician treatment recommendations made for patients within 120 days after NGS results became available. Physicians' post-test patient treatment recommendations were classified as targeted therapy, precision medicine clinical trial, immunotherapy, hormonal therapy, chemotherapy/radiation, surgery, transplant, or non-therapeutic (hospice, surveillance, or palliative care), and were compared to NGS report recommendations for agreement.

\section{Results \\ Patients}

Patients with 29 tumor types (Table 3) were tested, with breast cancer, colorectal carcinoma, lung carcinoma, melanoma, ovarian carcinoma, prostate cancer, and sarcoma being most common with each representing 5\% or more of the total tests. Median age of all patients tested (64) and gender (55\% males, $45 \%$ females) was within expectations for a cancer therapeutic management-focused test. Most patients had advanced (Stage III/IV) disease $(540 / 657$; 88\%), but for a patient subset, stage was not reported when the NGS test was ordered (77/ $657 ; 12 \%)$. The majority of tests $(76 \% ; 497 / 657)$ were for patients currently undergoing treatment and/or had at least one prior treatment when the NGS test was ordered. Repeat testing was mostly restricted to lung cancer patients with resistance to prior targeted therapies. 
Table 2 Level 1 and 2 evidence therapeutic variant associations tested by OmniSeq Comprehensive ${ }^{\oplus}$ (June 2017)

\begin{tabular}{|c|c|c|}
\hline \multicolumn{3}{|l|}{ Level 1 (Companion Diagnostics) } \\
\hline Genomic Variant(s) & Tumor Type & Therapy(ies) \\
\hline ALK fusion & Lung & alectinib, brigatinib, ceritinib, crizotinib \\
\hline BRAF V600E or BRAF V600K mutation & Melanoma & $\begin{array}{l}\text { cobimetinib + vemurafenib; dabrafenib; dabrafenib + trametinib; } \\
\text { trametinib; vemurafenib }\end{array}$ \\
\hline BRAF V600E or BRAF V600K mutation & Lung & dabrafenib + trametinib \\
\hline BRCA mutation & Ovarian & olaparib, rucaparib \\
\hline EGFR exon 19 deletion & Lung & afatinib; bevacizumab + erlotinib; erlotinib; gefitinib; osimertinib \\
\hline EGFR exon 20 insertion $^{a}$ & Lung & gefitinib \\
\hline EGFR G719 mutation & Lung & afatinib; gefitinib \\
\hline EGFR L858R mutation & Lung & afatinib; bevacizumab + erlotinib; erlotinib; gefitinib; osimertinib \\
\hline EGFR L861Q mutation & Lung & afatinib; gefitinib \\
\hline EGFR S768I mutation & Lung & afatinib; gefitinib \\
\hline EGFR T790 M mutation ${ }^{a}$ & Lung & gefitinib \\
\hline EGFR T790 M mutation & Lung & osimertinib \\
\hline ERBB2 amplification & Breast & $\begin{array}{l}\text { ado-trastuzumab emtansine; lapatinib + aromatase inhibitor; } \\
\text { lapatinib + chemo; lapatinib + trastuzumab; neratinib; } \\
\text { pertuzumab + trastuzumab + chemo; trastuzumab; } \\
\text { trastuzumab + aromatase inhibitor; trastuzumab + chemo }\end{array}$ \\
\hline ERBB2 amplification & Esophageal & trastuzumab; trastuzumab + chemo \\
\hline ERBB2 amplification & Gastric/GEJ & trastuzumab; trastuzumab + chemo \\
\hline KRAS A146 or KRAS A59 mutation ${ }^{a}$ & Colorectal & cetuximab; panitumumab \\
\hline KRAS exon 2, 3 or 4 mutation $^{a}$ & Colorectal & cetuximab; cetuximab + chemo; panitumumab + chemo \\
\hline KRAS G12, KRAS G13, KRAS K117, or KRAS Q61 mutation ${ }^{a}$ & Colorectal & cetuximab; panitumumab \\
\hline NRAS A146 or NRAS A59 mutation ${ }^{a}$ & Colorectal & cetuximab; panitumumab \\
\hline NRAS exon 2, 3 or 4 mutation $^{\text {a }}$ & Colorectal & cetuximab; cetuximab + chemo; panitumumab + chemo \\
\hline NRAS G12, NRAS G13, NRAS K117, or NRAS Q61 mutation ${ }^{a}$ & Colorectal & cetuximab; panitumumab \\
\hline ROS1 fusion & Lung & crizotinib \\
\hline \multicolumn{3}{|l|}{ Level 2 (Professional Practice Guidelines) } \\
\hline Genomic Variant & Tumor Type & Therapy \\
\hline ALK fusion & Sarcoma & ceritinib, crizotinib \\
\hline ALK fusion ${ }^{a}$ & Lung & EGFR tyrosine kinase inhibitor \\
\hline BRAF mutation & Thyroid & vemurafenib \\
\hline BRAF V600E mutation & Colorectal & $\begin{array}{l}\text { cetuximab + vemurafenib + chemo; panitumumab + } \\
\text { vemurafenib + chemo }\end{array}$ \\
\hline BRAF V600E mutation & Lung & dabrafenib; vemurafenib \\
\hline ERBB2 mutation & Lung & ado-trastuzumab emtansine \\
\hline ERBB2 mutation & Head and Neck & trastuzumab \\
\hline ERBB2 mutation & Breast & trastuzumab + chemo \\
\hline KIT exon 9 mutation & Sarcoma & imatinib \\
\hline KIT exon 11 mutation & Sarcoma & imatinib \\
\hline KIT exon 11 or KIT exon 13 mutation & Melanoma & imatinib \\
\hline KRAS mutation ${ }^{\mathrm{a}}$ & Lung & $\begin{array}{l}\text { EGFR tyrosine kinase inhibitor (afatinib; erlotinib; erlotinib; } \\
\text { gefitinib; osimertinib) }\end{array}$ \\
\hline MET amplification or MET exon 14 skipping mutation & Lung & crizotinib \\
\hline NF1 mutation ${ }^{a}$ & Sarcoma & imatinib \\
\hline PDGFRA D842V mutation ${ }^{a}$ & Sarcoma & dasatinib; imatinib \\
\hline
\end{tabular}


Table 2 Level 1 and 2 evidence therapeutic variant associations tested by OmniSeq Comprehensive ${ }^{\oplus}$ (June 2017) (Continued)

\begin{tabular}{lll}
\hline RET fusion & Lung & cabozantinib; vandetanib \\
RET mutation & Thyroid & vandetanib \\
ROS1 fusion & Lung & alectinib; ceritinib \\
ROS1 fusion & Lung & EGFR tyrosine kinase inhibitor (afatinib; erlotinib; erlotinib; \\
& & gefitinib; osimertinib) \\
\hline
\end{tabular}

${ }^{\text {a }}$ These mutations confer resistance to the associated therapeutic (contraindication)

NGS test results and physician treatment recommendations by FDA evidence level

A total of 2777 genomic alterations were identified in 657 tests with an average of 4.2 mutations per test. Frequently identified mutations were level 3 (1532; 55\%), followed by VUTS (1045; 38\%), level 2 (107; 4\%), and level $1(93 ; 3 \%)$.

\section{Level 1 variants}

A total of 92 variants with companion diagnostic level 1 supporting evidence as the most actionable result were detected in $14 \%(89 / 657)$ of tests as the most actionable evidence across 5 tumor types (Table 4). For the majority of these tests $(94 \% ; 84 / 89)$, a single genomic alteration was identified, with and no more than two level 1 variants reported in the remaining 5 tests. All tests with a level 1 variant had at least one other level 2 or level 3 alteration. The most frequent level 1 variants detected were identified in KRAS (colorectal cancer), EGFR (lung cancer), and $B R A F$ (melanoma). Additional tumor types with at least one level 1 variant detected included breast and ovarian cancer.

The most common physician treatment recommendation for tests with level 1 evidence variant results was targeted therapy for $(26 / 89 ; 29 \%)$ of tests (48\% excluding mutations with contraindications: KRAS, NRAS), followed by chemotherapy/radiation for $23 \%$. The majority of these patients initiated chemotherapy/radiation prior to the test being performed with the presumed intention of planning for future treatment. Immunotherapy was a frequent recommendation (14/89; 16\%), related to lack of EGFR or $A L K$ alterations in NSCLC. Non-therapeutic recommendations (hospice or palliative care) were infrequent when variants with level 1 evidence were detected ( $2 \%$ of tests). Clinical trial recommendations were also uncommon (5/89; 6\%) (Fig. 1).

\section{Level 2 variants}

A total of 109 variants with level 2 professional practice guideline supporting evidence for targeted therapy as the most actionable findings were detected in 100/657 (15\%) of tests across five tumors types (colorectal carcinoma, lung cancer, melanoma, sarcoma, thyroid carcinoma). Similar to tests with a level 1 results, the majority of these tests $(95 / 100 ; 97 \%)$ documented a single level 2 variant, and no more than two level 2 variants were reported for any given test. All tests with a level 2 variant also harbored at least one level 3 alteration. The most frequent level 2 variants were KRAS mutations in lung cancer. The second most frequent level 2 results encompassed atypical activating EGFR mutations in lung cancer patients, supporting use of EGFR inhibitors for certain alterations. The remaining level 2 variant results were distinct from level 1 results, and included a variety of alterations, all at a prevalence $<5 \%$ for that particular tumor type or a single case result (Table 4).

The most frequent physician treatment recommendation in the presence of level 2 evidence was chemotherapy/radiation $(32 / 100 ; 32 \%)$, followed by immunotherapy for $25 /$ 100 tests (25\%). Targeted therapy was a less frequent recommendation $(11 / 98 ; 11 \%)$ with non-therapeutic recommendations being slightly more frequent (14/100; $14 \%)$. Clinical trial recommendations were also uncommon in this group (2/100; 2\%) (Fig. 1).

\section{Level 3 variants}

There were 419 tests (65\%) with level 3 genomic alterations as the most actionable variants. These results supported potential enrollment in either precision medicine clinical trials or use of off-label therapy, and were the most common result overall: mutations (1532/2777; $55 \%)$, tests $(579 / 657 ; 88 \%)$, and all genes tested $(67 / 144$; $47 \%)$. Genes implicated by a level 3 mutation in at least $5 \%$ of all tests included TP53, ATM, CDKN2A, BRCA2, PTEN, BRCA1, PIK3CA, TSC2, KDR, NF1, MET, RB1, and $P T C H 1$ in descending order of prevalence (Table 5).

For the 419 tests for which level 3 variants were identified as the most actionable, the rate of clinical trial treatment recommendations by physicians was $7 \%(28 /$ 419). Commonly recommended in this group was chemotherapy/radiation (193/419; 46\%), followed by immunotherapy $(49 / 419 ; 11 \%)$. Targeted therapy recommendations were uncommon $(15 / 419 ; 4 \%)$. Non-therapeutic options were recommended to $10 \%(41 / 419)$ of the cases belonging to this group, and not remarkably different from patients with a level 2 result (14\%) (Fig. 1).

Overall, a total of 111 mutations with off-label indications were identified in 90 tests with no level 1 or 2 evidence. The majority of these off-label indications were BRCA1 or BRCA2 mutations $(90 / 111 ; 81 \%)$, which were documented across 20 different tumor types. Less frequent mutations linked to off-label indications included 
Table 3 Tested patient characteristics $(n=646)$

\begin{tabular}{|c|c|}
\hline \multicolumn{2}{|l|}{$\overline{\text { Age }}$} \\
\hline Median, yrs & 64 \\
\hline Range, yrs & $6-93$ \\
\hline \multicolumn{2}{|l|}{ Gender, n (\%) } \\
\hline Female & $359(55 \%)$ \\
\hline Male & $298(45 \%)$ \\
\hline \multicolumn{2}{|l|}{ Stage, n (\%) } \\
\hline Stage I & $9(1.4 \%)$ \\
\hline Stage \| & $11(1.7 \%)$ \\
\hline Stage III & $54(8 \%)$ \\
\hline Stage IV & $506(77 \%)$ \\
\hline Unknown & $77(12 \%)$ \\
\hline \multicolumn{2}{|l|}{ Tumor Type, n (\%) } \\
\hline Bladder & $4(0.61 \%)$ \\
\hline Brain & $15(2.3 \%)$ \\
\hline Breast & $34(5 \%)$ \\
\hline Cervical & $4(0.61 \%)$ \\
\hline Colorectal & $68(10 \%)$ \\
\hline Endocrine & $3(0.46 \%)$ \\
\hline Endometrial & $25(3.8 \%)$ \\
\hline Esophageal & $13(2 \%)$ \\
\hline Eye & $1(0.2 \%)$ \\
\hline Female Genital & $1(0.2 \%)$ \\
\hline Gallbladder & $3(0.5 \%)$ \\
\hline Head and Neck & $4(0.6 \%)$ \\
\hline Kidney and Renal Pelvis & $10(1.5 \%)$ \\
\hline Liver and Bile Duct & $1(0.15 \%)$ \\
\hline Lung & 205 (31\%) \\
\hline Melanoma & $51(8 \%)$ \\
\hline Mesothelioma & $4(0.6 \%)$ \\
\hline Neuroendocrine Tumors & $14(2.1 \%)$ \\
\hline Non-Melanoma Skin & $2(0.3 \%)$ \\
\hline Ovarian & $44(7 \%)$ \\
\hline Pancreatic & $9(1.4 \%)$ \\
\hline Prostate & $34(5 \%)$ \\
\hline Sarcoma & $87(13 \%)$ \\
\hline Small Intestine & $3(0.5 \%)$ \\
\hline Stomach & $3(0.5 \%)$ \\
\hline Testicular & $1(0.2 \%)$ \\
\hline Thymic & $3(0.5 \%)$ \\
\hline Thyroid & $4(0.6 \%)$ \\
\hline Unknown Primary & $7(1 \%)$ \\
\hline \multicolumn{2}{|l|}{ Prior systemic treatment } \\
\hline Unknown & $4(0.6 \%)$ \\
\hline 0 & 156 (24\%) \\
\hline $1+$ & 497 (76\%) \\
\hline
\end{tabular}

Table 4 Frequency of detected variants by FDA level of supporting evidence (as of June 2017)

Tests with variants supported by level 1 evidence as the most actionable $(n=89)$

Gene Tumor Number of Detected Variants Type $\quad$ Variants $(n=92)$

KRAS Colorectal 32

EGFR Lung 17

BRAF Melanoma 17

BRCA1 Ovarian 7

BRAF Lung 5

ALK Lung 5

NRAS Colorectal 3

BRCA2 Ovarian 3

ERBB2 Breast 2

ROS1 Lung 1

Codon $12,13,61,146$ single nucleotide variants

L858R, T790 M, exon 19 deletions

V600E, V600K

Multiple single nucleotide variants \& indels*

V600E

ALK (EML4) fusion

Codon 12 \& 61 single nucleotide variants

Multiple single nucleotide variants*

HER-2 amplification

ROS1 (EZR) fusion

Tests with variants supported by level 2 evidence as the most actionable $(n=100)$

Gene Tumor Number of Detected Detected Mutations Type Variants $(n=109)$

KRAS Lung 71

Codon $12,13,61,146$ single nucleotide variants \& insertions

EGFR Lung 9

Atypical exon 19 indels, exon 20 insertions, G719C, S768I, L8610

MET Lung 7

MET amplification and exon 14 skip

Multiple single nucleotide variants*

Multiple exon 11 single nucleotide variants

ERBB2 Lung 3

KIT Sarcoma 2 (GIST)

BRAF Colorectal 2

RET Thyroid 1

E365K, Y742_A745dup

Exon 9 and 11 single nucleotide variants

V600E

M918T

RET Lung 1

RET (KIF5B) fusion

KRAS Colorectal 1

V14I

NRAS Colorectal 1

Q61R

KIT Sarcoma 1

V559D (exon 11)

ALK Sarcoma 1

ALK (TPM3) fusion

activating single nucleotide variants or indels (insertions/deletions) in ERBB2 (HER-2), KIT, BRAF, and RET, as well as $M E T$ copy number gain or exon 14 skip. Off-label targeted therapy recommendations were infrequent $(11 / 111 ; 10 \%)$. 


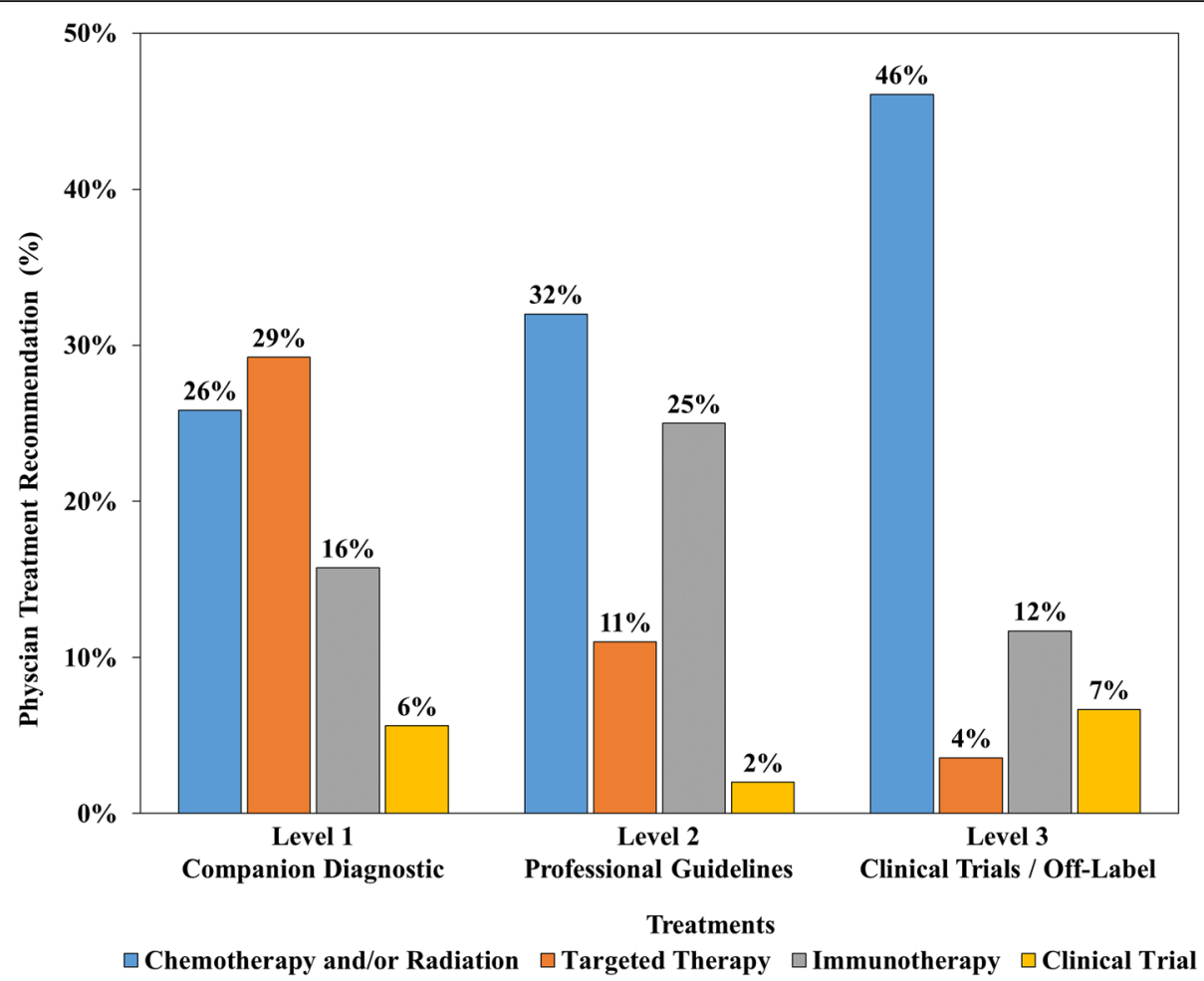

Fig. 1 Physician treatment recommendations by the highest level of supporting clinical evidence to treat by targeted therapy for each test. Physician treatment recommendations for targeted therapies were most frequent for patient tests detecting variants supported by level 1 companion diagnostic evidence for targeted therapy (26/89; 29\%), followed by tests with variants supported by level 2 practice guideline evidence as the most actionable result (11/100; 11\%), and tests with variants supported by level 3 clinical trial/off-label evidence as the most actionable result (15/419; 4\%). Recommendations for chemotherapy and/or radiation were more common for tests with variants supported by level 3 evidence (193/419; 46\%) than for patient test results with level $2(32 / 100 ; 32 \%)$ or level $1(23 / 89 ; 26 \%)$ evidence. Recommendations for clinical trials were infrequent for test results across all 3 evidence levels for patients with companion diagnostic level 1 (5/89; 6\%), level 2 (2/100; 2\%), and level 3 (28/419; 7\%) evidence. Recommendations for immunotherapy were relatively frequent for tests with targeted therapy level 1 (14/89; 16\%), level 2 (25/100; 25\%) or level 3 (49/419; 12\%) supporting evidence

\section{VUTS}

For a subset of tests (49/657; 7\%), no variants were classified as having clinical significance based on FDA variant classification guidance. It is worthy to note that this group had the highest recommendation rates for chemotherapy/radiation $(26 / 49 ; 53 \%)$, as well as and non-therapeutic options $(8 / 49 ; 16 \%)$ compared to tests with variants supported by level 1,2 or 3 evidence. Targeted therapy was never recommended in this group.

\section{Tumor type-specific results}

Of 29 tumor types tested, 24 did not harbor level 1 or 2 variants. These included mesotheliomas, sarcomas, bladder, brain, cervical, endocrine, endometrial, gastroesophageal, eye, genital, gallbladder, head and neck, kidney, renal pelvis, liver, bile duct, neuroendocrine, non-melanoma skin, pancreatic, prostate, small bowel, stomach, testicular, thymic, thyroid, and unknown primary cancers. Most of these tumor types also did not have biomarker-directed indications approved for targeted therapy, either on label or in professional practice guidelines. None of the 34 prostate cancers tested expressed a level 1 or 2 alteration. Of the 24 tumor types with no actionable level 1 or 2 variants, only 2 (gastroesophageal carcinomas and gastrointestinal stromal tumors) harbored mutations that met the list of genomic variants identified as therapeutic association with a high level of evidence (Table 4).

The remaining 5 tumor types with at least one level 1 or 2 variant included melanoma, breast, colorectal, lung, and ovarian cancers accounted for 61\% (402/657) of the total tests performed, and do have biomarker-directed indications for targeted therapy, accurately reflecting the prevalence of these tumors and their current position in the precision medicine hierarchy per oncologist test usage.

\section{Discussion}

This study demonstrates the application of FDA-guidance for NGS variant classification in oncology therapeutic management, and compares the test results to treatment recommendations the way healthcare providers typically 
Table 5 Level 3 variant frequency by gene and number of tests

\begin{tabular}{|c|c|c|c|}
\hline Gene & Number of Variants & Number of Tests & $\%$ of Tests \\
\hline TP53 & 310 & 280 & 0.43 \\
\hline ATM & 158 & 139 & 0.21 \\
\hline CDKN2A & 96 & 91 & 0.14 \\
\hline BRCA2 & 101 & 90 & 0.14 \\
\hline PTEN & 85 & 74 & 0.11 \\
\hline BRCA1 & 69 & 61 & 0.09 \\
\hline PIK3CA & 63 & 56 & 0.09 \\
\hline TSC2 & 45 & 44 & 0.07 \\
\hline KDR & 43 & 41 & 0.06 \\
\hline NF1 & 50 & 41 & 0.06 \\
\hline MET & 37 & 37 & 0.06 \\
\hline RB1 & 37 & 37 & 0.06 \\
\hline PTCH1 & 37 & 34 & 0.05 \\
\hline STK11 & 30 & 28 & 0.04 \\
\hline FBXW7 & 25 & 25 & 0.04 \\
\hline NF2 & 24 & 24 & 0.04 \\
\hline NRAS & 23 & 23 & 0.04 \\
\hline APC & 23 & 22 & 0.03 \\
\hline TSC1 & 23 & 21 & 0.03 \\
\hline KRAS & 19 & 18 & 0.03 \\
\hline IDH1 & 18 & 17 & 0.03 \\
\hline CDK4 & 18 & 17 & 0.03 \\
\hline CSF1R & 15 & 14 & 0.02 \\
\hline EGFR & 14 & 12 & 0.02 \\
\hline BRAF & 13 & 11 & 0.02 \\
\hline ERBB2 & 15 & 11 & 0.02 \\
\hline FGFR3 & 12 & 10 & 0.02 \\
\hline FGFR1 & 10 & 9 & 0.01 \\
\hline AKT1 & 8 & 8 & 0.01 \\
\hline SMO & 9 & 8 & 0.01 \\
\hline $\mathrm{MSH} 2$ & 9 & 6 & 0.01 \\
\hline CCND1 & 6 & 6 & 0.01 \\
\hline KIT & 7 & 6 & 0.01 \\
\hline PIK3R1 & 6 & 6 & 0.01 \\
\hline RET & 6 & 6 & 0.01 \\
\hline SMARCB1 & 5 & 4 & 0.01 \\
\hline CHEK2 & 4 & 4 & 0.01 \\
\hline CCNE1 & 4 & 4 & 0.01 \\
\hline MYC & 4 & 4 & 0.01 \\
\hline BAP1 & 4 & 4 & 0.01 \\
\hline ERBB4 & 3 & 3 & $<.01$ \\
\hline DDR2 & 3 & 3 & $<.01$ \\
\hline FLT3 & 3 & 3 & $<.01$ \\
\hline MAP2K1 & 3 & 3 & $<.01$ \\
\hline
\end{tabular}

Table 5 Level 3 variant frequency by gene and number of tests (Continued)

\begin{tabular}{|c|c|c|c|}
\hline Gene & Number of Variants & Number of Tests & $\%$ of Tests \\
\hline ALK & 3 & 3 & $<.01$ \\
\hline ESR1 & 2 & 2 & $<.01$ \\
\hline GNA11 & 2 & 2 & $<.01$ \\
\hline ERBB3 & 2 & 2 & $<.01$ \\
\hline HRAS & 2 & 2 & $<.01$ \\
\hline ABL1 & 2 & 2 & $<.01$ \\
\hline JAK2 & 2 & 2 & $<.01$ \\
\hline MTOR & 2 & 2 & $<.01$ \\
\hline MYCL & 2 & 2 & $<.01$ \\
\hline NFE2L2 & 2 & 2 & $<.01$ \\
\hline NTRK1 & 2 & 2 & $<.01$ \\
\hline MAPK1 & 2 & 2 & $<.01$ \\
\hline AR & 1 & 1 & $<.01$ \\
\hline CDK6 & 1 & 1 & $<.01$ \\
\hline FGFR2 & 1 & 1 & $<.01$ \\
\hline JAK1 & 1 & 1 & $<.01$ \\
\hline WT1 & 1 & 1 & $<.01$ \\
\hline MDM2 & 1 & 1 & $<.01$ \\
\hline NTRK3 & 1 & 1 & $<.01$ \\
\hline RAF1 & 1 & 1 & $<.01$ \\
\hline SRC & 1 & 1 & $<.01$ \\
\hline $\mathrm{IDH} 2$ & 1 & 1 & $<.01$ \\
\hline
\end{tabular}

do (i.e., retrospectively) to obtain reimbursements. In particular, this study provides evidence from a current clinical practice where physicians formulate treatment recommendations based on a cancer-focused comprehensive NGS assay in the context of new guidelines from FDA. One conclusion from this study is that physicians do not recommend (or at least do not document recommending) clinical trials as often as they are indicated by NGS testing. We recognize that capturing treating physicians clinical trial recommendations solely by medical record review may generate an underestimation of the actual physician intent (i.e. patient ineligibility based on pre-screening evaluation) or patient-physician interaction in which the highest level of evidence is associated with an investigational study (level 3 evidence). In the group of tests with no on-label indications for targeted therapy, chemotherapy/radiation were the most frequent recommendations, suggesting that traditional approaches to cancer are still highly regarded by both oncologists and patients, or at least are still regarded as the most viable option given all the complex factors that contribute to decision making. This study also suggests that physicians are using NGS results appropriately to recommend targeted therapy. Targeted therapy was recommended for $48 \%$ of tests with 
level 1 evidence, $11 \%$ of tests with level 2 evidence, 3.6\% of tests with level 3 evidence, and never in the presence of VUTS. This may have been positively influenced by the fact that testing and recommendations were performed in a NCI-designated comprehensive cancer center with access to a molecular tumor board, which can aid in treatment decision recommendations.

The majority of tumor types tested in this study have no level 1 or 2 therapeutic drug associations, implying that maximal benefit could originate from either a clinical trial or an off-label recommendation, both of which were infrequently recommended by treating physician, at least during the data collection period in this study. There is doubt that following such patients in a registry will provide any benefit when physicians do not strongly support enrollment in clinical trials but generally recommend chemotherapy/radiation. One factor that may offset such a perceived lack of benefit from NGS testing for targeted therapy is immunotherapy. Immunotherapy was recommended by physicians in 16\% of tests with level 1 evidence, $25 \%$ of tests with level 2 evidence, $12 \%$ of tests with level 3 evidence, and $6 \%$ of tests documenting VUTS. This suggests that physicians are often using NGS results to exclude targeted therapy prior to recommending immunotherapy.

Many centers in the US are implementing clinical oncology pathways and collecting evidence as we did, which may be more accurate and representative than the current approach of multiple laboratories. Such pathways should aim at aligning care to national guidelines or, when appropriate, define the rationale for deviation from guidelines [12]. Another key objective for these pathways should be to support clinical trials, and to identify the barriers to enrollment. Some pathway programs require review of relevant clinical trials as the first treatment choice before allowing selection of "standard" therapies [13]. Pathway systems also provide a registry that identifies patients starting a new cancer treatment, what that treatment is, if the patient accepted to join a clinical trial, and how treatment compares to national guidelines. Pathway systems may provide the basis for proper identification of patients eligible for NGS and related clinical trials. Databases generated in the context of these pathways may also provide a robust foundation for the required $\mathrm{Na}-$ tional Institute of Health Genetic Testing Registry (NIH GTR) as per the new FDA guidelines for NGS.

There are multiple limitations to the present study, many of which related to the fact that it was retrospective and involved no direct interaction with physicians other than test ordering, test reporting, or molecular tumor board review. Recommendations were captured from clinical documentation which may be incomplete (e.g. not specifically documenting that a patient with is not eligible for clinical trial due to exclusion criteria) and may not record past discussions with ordering physicians. This may partially explain the low documented rate of recommendations to clinical trials, which may not fully represent the actual clinical practice. Eligibility for a clinical trial often includes multiple factors beyond molecular parameters, such as number and types of prior therapies, comorbidities, etc. Physician recommendations were captured within 120 days post-NGS test for patients that had at least 60 days of follow up time. Several clinical trials, such as the NCI-MATCH, require that patients have explored standard therapeutic options first and recommendation for a clinical trial may occur much later in a patient's treatment course, often after multiple episodes of care.

\section{Conclusions}

NGS is a well-established technology, yet it lacks standardized regulatory approval. The majority of tumors do not have on-label, variant-directed targeted therapy indications, however, our data show that physicians generally understand NGS results and apply them appropriately, and that the FDA has provided valid guidance a pathway for medical policy coverage for at least a subset of tumor types. As precision medicine advances toward regulatory approval in the standard of care setting, coverage by both the Centers for Medicaid and Medicare (CMS) and other payers will require a consistent reproducible method of presenting evidence.

\section{Abbreviations \\ ACMG: American College of Medical Genetics and Genomics; CAP: College of American Pathologists; CLIA: Clinical Laboratory Improvement Amendments; EMA: European Medical Association; ESMO: European Society for Medical Oncology; FDA: Food and Drug Administration; GTR: Genetic Testing Registry; NCBI: National Center for Biotechnology Information; NCCN: National Comprehensive Cancer Network; NCI-MATCH: National Cancer Institute's Molecular Analysis for Therapy Choice.; NGS: Next generation sequencing; $\mathrm{NIH}$ : National Institute of Health; NYS CLEP: New York State Clinical Laboratory Evaluation Program; NYSDOH: New York State Department of Health; OCP: OmniSeq Comprehensive ${ }^{\oplus}$; RPCCC: Roswell Park Comprehensive Cancer Center}

\section{Acknowledgements}

Not Applicable

\section{Funding}

OmniSeq, Inc. (Buffalo, NY) funded the design of the study; the collection, analysis, and interpretation of data; and the writing of the manuscript.

\section{Availability of data and materials}

The data that support the findings of this study are available from OmniSeq, Inc. but restrictions apply to the availability of these data, which were used under license for the current study, and so are not publicly available. Data are however available from the authors upon reasonable request and with permission of Omniseq, Inc..

\section{Authors' contributions}

GD, MLV, AE, HC, AGM, PB, SE, SA, MO, GC, KO, KK, PF, SL, BK, CM, STG, JMC, and MSE contributed to the design and conduct of the study. SP, JMC, MKN STG, APS, BB, JA, VG, HTD, MQ, YW, FLL, and CM contributed to the design and conduct of the study collection, management, analysis, and interpretation of the data. All authors contributed to the preparation, review, and approval of the final manuscript and the decision to submit the manuscript for publication. 


\section{Ethics approval and consent to participate}

OmniSeq's analysis utilized deidentified data that was considered non-human subjects research under IRB-approved protocol (BDR \#073166) at Roswell Park Comprehensive Cancer Center (Buffalo, NY).

\section{Consent for publication}

Not applicable

\section{Competing interests}

Disclosures: SP, JMC, MKN, STG, APS, BB, JA, VG, HTD, MQ, YW, FLL, and CM are all employees of OmniSeq, Inc. (Buffalo, NY) and hold restricted stock in OmniSeq, Inc. GD, MLV, AE, HC, AGM, PB, SE, SA, MO, GC, KO, KK, PF, SL, BK, CM, STG, JMC, and MSE are employees of Roswell Park Comprehensive Cancer Center (Buffalo, NY). Roswell Park Comprehensive Cancer Center is the majority shareholder of OmniSeq, Inc. MSE and LG provide remunerated consulting to OmniSeq, Inc.

\section{Publisher's Note}

Springer Nature remains neutral with regard to jurisdictional claims in published maps and institutional affiliations.

\section{Author details}

${ }^{1}$ Department of Medicine, Roswell Park Comprehensive Cancer Center, Buffalo, NY 14263, USA. ${ }^{2}$ OmniSeq, Inc., Buffalo, NY 14203, USA. ${ }^{3}$ Department of Pathology, Roswell Park Comprehensive Cancer Center, Buffalo, NY 14263, USA. ${ }^{4}$ Center for Personalized Medicine, Roswell Park Comprehensive Cancer Center, Buffalo, NY 14263, USA. ${ }^{5}$ Cancer Genetics and Genomics, Roswell Park Comprehensive Cancer Center, Buffalo, NY 14263, USA. ${ }^{6}$ Division of Gynecologic Oncology, Roswell Park Comprehensive Cancer Center, Buffalo, NY 14263, USA. ${ }^{7}$ Department of Radiation Oncology, Weill Cornell Medical College, New York, NY 10065, USA. ${ }^{8}$ Sandra and Edward Meyer Cancer Center, New York, NY 10065, USA. ' Université Paris Descartes/Paris V, Paris, France. ${ }^{10}$ Department of Surgery, Roswell Park Comprehensive Cancer Center, Buffalo, NY 14263, USA.

Received: 18 July 2018 Accepted: 10 January 2019

Published online: 18 January 2019

\section{References}

1. U.S. Food and Drug Administration. CDRH'S APPROACH TO TUMOR PROFILING NEXT GENERATION SEQUENCING TESTS. Silver Spring, MD: U.S. Food and Drug Administration; 2017. https:/www.fda.gov/downloads/ MedicalDevices/ProductsandMedicalProcedures/InVitroDiagnostics/ UCM584603.pdf.

2. FDA. EVALUATION OF AUTOMATIC CLASS III DESIGNATION FOR MSK-IMPACT (Integrated Mutation Profiling of Actionable Cancer Targets). 2017;:1-57. doi:https://doi.org/10.1164/rccm.201205-08150C.

3. Tsimberidou A-M, Iskander NG, Hong DS, Wheler JJ, Falchook GS, Fu S, et al. Personalized medicine in a phase I clinical trials program: the MD Anderson Cancer Center initiative. Clin Cancer Res. 2012;18:6373-83. https://doi.org/10. 1158/1078-0432.CCR-12-1627.

4. Stockley TL, Oza AM, Berman HK, Leighl NB, Knox JJ, Shepherd FA, et al. Molecular profiling of advanced solid tumors and patient outcomes with genotype-matched clinical trials: the Princess Margaret IMPACT/COMPACT trial. Genome Med. 2016;8:109. https://doi.org/10.1186/s13073-016-0364-2.

5. Arango NP, Brusco L, Shaw KRM, Chen K, Eterovic AK, Holla V, et al. A feasibility study of returning clinically actionable somatic genomic alterations identified in a research laboratory. Oncotarget. 2017:8:41806-14. https:/doi. org/10.18632/oncotarget.16018.

6. Hilal T, Nakazawa M, Hodskins J, Villano JL, Mathew A, Goel G, et al. Comprehensive genomic profiling in routine clinical practice leads to a low rate of benefit from genotype-directed therapy. BMC Cancer. 2017; 17:602. https://doi.org/10.1186/s12885-017-3587-8.

7. Jordan EJ, Kim HR, Arcila ME, Barron D, Chakravarty D, Gao J, et al. Prospective comprehensive molecular characterization of lung adenocarcinomas for efficient patient matching to approved and emerging therapies. Cancer Discov. 2017;7: 596-609. https://doi.org/10.1158/2159-8290.CD-16-1337.

8. Sireci AN, Aggarwal VS, Turk AT, Gindin T, Mansukhani MM, Hsiao SJ. Clinical genomic profiling of a diverse Array of oncology specimens at a large academic Cancer center. J Mol Diagnostics. 2017;19:277-87. https://doi.org/ 10.1016/j.jmoldx.2016.10.008.
9. Tsimberidou A-M, Hong DS, Ye Y, Cartwright C, Wheler JJ, Falchook GS, et al. Initiative for molecular profiling and advanced Cancer therapy (IMPACT): an MD Anderson precision medicine study. JCO Precis Oncol. 2017;2017:1-18. https://doi.org/10.1200/PO.17.00002.

10. Schram AM, Reales D, Galle J, Cambria R, Durany R, Feldman D, et al. Oncologist use and perception of large panel next-generation tumor sequencing. Ann Oncol. 2017;28:2298-304. https://doi.org/10.1093/annonc/mdx294.

11. Green RC, Berg JS, Grody WW, Kalia SS, Korf BR, Martin CL, et al. ACMG recommendations for reporting of incidental findings in clinical exome and genome sequencing. Genet Med. 2013;15:565-74. https://doi.org/10.1038/ gim.2013.73.

12. Zon RT, Edge SB, Page RD, Frame JN, Lyman GH, Omel JL, et al. American Society of Clinical Oncology criteria for high-quality clinical pathways in oncology. J Oncol Pract. 2017;13:207-10. https://doi.org/10.1200/JOP.2016. 019836

13. Daly B, Zon RT, Page RD, Edge SB, Lyman GH, Green SR, et al. Oncology Clinical Pathways: Charting the Landscape of Pathway Providers. J Oncol Pract. 2018;14:e194-200. https://doi.org/10.1200/JOP.17.00033.

\section{Ready to submit your research? Choose BMC and benefit from:}

- fast, convenient online submission

- thorough peer review by experienced researchers in your field

- rapid publication on acceptance

- support for research data, including large and complex data types

- gold Open Access which fosters wider collaboration and increased citations

- maximum visibility for your research: over $100 \mathrm{M}$ website views per year

At BMC, research is always in progress.

Learn more biomedcentral.com/submissions 